\title{
Natural generalization of the ground-state Slater determinant to more than one dimension
}

\author{
D. K. Sunkd \\ Department of Physics, Faculty of Science, University of Zagreb, \\ Bijenička cesta 32, HR-10000 Zagreb, Croatia.
}

\begin{abstract}
The basic question is addressed, how the space dimension $d$ is encoded in the Hilbert space of $N$ identical fermions. There appears a finite number $N !^{d-1}$ of many-body wave functions, called shapes, which cannot be generated by trivial combinatorial extension of the one-dimensional ones. A general algorithm is given to list them all in terms of standard Slater determinants. Conversely, excitations which can be induced from the one-dimensional case are bosonised into a system of distinguishable bosons, called Euler bosons, much like the electromagnetic field is quantized in terms of photons distinguishable by their wave numbers. Their wave functions are given explicitly in terms of elementary symmetric functions, reflecting the fact that the fermion sign problem is trivial in one dimension. The shapes act as vacua for the Euler bosons. They are the natural generalization of the single-Slater-determinant form for the ground state to more than one dimension. In terms of algebraic invariant theory, the shapes are antisymmetric invariants which finitely generate the $N$-fermion Hilbert space as a graded algebra over the ring of symmetric polynomials. Analogous results hold for identical bosons.
\end{abstract}

PACS numbers: 03.65.Ta, 03.65.Fd, 31.15.-p

\section{INTRODUCTION}

Quantum effects are sometimes counter-intuitive because physics happens in the space of wave functions, not in the geometrical "laboratory" space of Newtonian mechanics. Conversely, molecular isomerism, the phenomenon that a given set of identical atoms can arrange itself in molecules of different shapes, is quite intuitive geometrically. The question arises, how is it manifested in wave-function space. The discreteness of wave functions must somehow limit the relative positions in laboratory space. In particular, one would like to have a qualitative argument, which shapes are possible solutions of the many-body Schrödinger equation, without a full calculation.

The choice of ground-state wave function - i.e. particular shape among possible isomers - is evidently related to the choice of a wave function with pronounced correlations, or collectivity. The notion of collectivity is usually taken to mean that the energy cannot be expressed as the sum of energies of single-particle wave functions. That intuition cannot be literally true, because the Kohn-Sham theorem [1] shows that it is possible to construct artificial single-particle states precisely by the requirement that the exact ground state energy can be expressed in this way.

Since Dirac introduced them 2, Slater determinants 3 have been the only fundamental antisymmetric forms available to construct optimized wave functions. Being a complete basis for the N-body Hilbert space, they encourage a functional-analytic, essentially structureless, view of that space, as a vector space in which the ground

\footnotetext{
* dks@phy.hr
}

state is just one particular linear combination of Slater determinants among many.

In particular, the Kohn-Sham method [1] is a special search in coefficient space, constrained by the requirement that the final linear combination can be written as a single Slater determinant in some new single-particle wave functions. However, the restriction to a Slaterdeterminant form is arbitrary, basically due to a lack of a priori alternatives. The price paid for it is that the new single-particle wave functions are artificial, even if the ground-state energy is correct. If the restriction is relaxed, the lack of structure in the functional-analytic approach (one set of $c$-numbers is in principle as good as another) leads to the impression that there is an infinity of possibilities to choose from.

Motivated by the above considerations, the present work explores a related but more qualitative idea of collectivity, based on wave-function properties rather than energies. If the single-particle wave functions are separable in the Cartesian coordinates of laboratory space, good candidates for many-body collective states should not inherit this separability. Such states are multidimensional in some non-trivial way, which is given a rigorous meaning here.

The main result is that there exist precisely $N !^{d-1}$ antisymmetric forms, called shapes, which are the basic building blocks of any antisymmetric $N$-body wave function in $d$ dimensions. This result is rooted in the algebraic theory of invariants [4, indeed it is expected in that context (known as "Hilbert's 14th problem"). However, it is unexpected to physicists and chemists, who are trained in the functional-analytic rather than algebraic approach to Hilbert spaces. In the former case, Hilbert space is viewed as a vector space spanned by an infinity of Slater determinants $\Psi_{i}$, in which any wave function may be 
written as

$$
\Psi=\sum_{i} c_{i} \Psi_{i}
$$

where the $c_{i}$ are $c$-numbers. When a vector space is endowed with an additional vector multiplication operation, it becomes an algebra. When the vectors are complex functions, the natural vector multiplication is just ordinary multiplication of functions. The switch to the algebraic approach is thus technically manifested as a generalization of the $c_{i}$ to symmetric functions of the space coordinates. Then it turns out that the sum becomes finite: many-body Hilbert space is a finitedimensional algebra. Only a finite number of antisymmetric forms $\Psi_{i}$ is needed to generate the whole Hilbert space, now viewed as a graded algebra over the ring of symmetric polynomials (for $N$ finite). These forms $\Psi_{i}$ are just the generators of the Hilbert-space algebra, called shapes here. In one dimension, there is only one shape, which is the ground-state Slater determinant. Thus shapes are generalizations of this particular Slater determinant to more than one dimension. They can always be expressed as superpositions of Slater determinants, because the latter are a complete basis. The connections between the algebraic and functional-analytic points of view for the physical $N$-body problem have only begun to be explored in the present work.

The shapes extend the notion of a vacuum state in an explicit and formally rigorous sense: the usual groundstate energy shift $\exp \left(-\beta E_{g s}\right) \equiv q^{E_{g s}}$ in the partition function is replaced by a shape polynomial $P(q)$, which counts all possible antisymmetric forms which can play the role of a vacuum. It is the generating function of the shapes. An algorithm is provided which generates the Hilbert-space span of all shapes in terms of Slater determinants. It may be interpreted as a machine-assisted way to generate ansätze for correlated ground states when $d>1$.

Excitations of any one of these vacuum states are described by the symmetric-function coefficients, in other words they are bosonic. In contrast to the shapes, they can be extended from $d=1$ as if the space directions were color labels, combined in all possible ways. These symmetric states are called Euler bosons, because their partition function was first obtained by Euler [5]. Euler bosons cannot exist by themselves. Each wave function in the scheme is based on some single shape, with or without an arbitrary number of excitations (Euler bosons) on top of it. In brief, the shapes represent all possible many-body vacua for the Euler bosons.

The classification in the present form does not include spin, and refers to only one kind of particle. Neither is an essential limitation. Including spin and different kinds of particles amounts to combining several generating algorithms of the type introduced here multiplicatively, which is unnecessary for an initial description. It is possible to think of the states here as referring to a concrete system, such as entangled atoms [6], or electrons in a quantum dot [7, 8, or in the vacancy of an electride [9, but an important aspect of the results is their abstraction and generality, based on a topological (node-counting) classification of wave functions, which is universal. In fact the basic algorithm operates at the level where single-particle wave functions are represented by formal powers, so that a term like $t^{k}$ refers to Hermite polynomials $H_{k}(x)$ in one realization and to standing waves $\sin (k+1) x$ in another. No result depends on the particular realization.

The article consists of two parts. The first is a selfcontained derivation of all the results in an abstract setting. The basic counting result is established, with a recursion for the shape polynomial. The one-dimensional case is solved in terms of the Euler bosons. A polynomial deflation algorithm is introduced to express the Eulerboson wave functions in terms of standard Slater determinants. This algorithm is used in $d>1$ to represent all trivial (separable) states in an ordered succession of subspaces, finding the shapes as the remainder (orthogonal complement) at each level. The second part consists of examples and illustrations. For $N=3$ particles in $d=2$ dimensions, all the six shapes are constructed step by step. Some numerical experiments are performed with the Coulomb interaction, to check that the scheme is not unstable with respect to it. Variational functions and simulations are discussed, with a minimal example.

While all the main formulas refer to fermions, in general the results for identical bosons are very similar. This may have direct repercussions for systems of entangled atoms. The bosonic case is compared to the fermionic one at the end, before the discussion and conclusions. Some textbook mathematics is collected in the appendices to make the article better self-contained.

\section{THE BASIC COUNTING RESULT}

\section{A. Partition function}

In physics, the partition function, or sum over states, is typically used in the context of thermodynamics, with the idea that each "state" being counted is thermodynamically possible, in the sense that it is an energy level of the actual system under consideration. In the present work, a more general approach is taken, where a "state" is simply any wave function, irrespective of whether there exists a Hamiltonian of which it is an eigenfunction. The only requirement on the partition function is that it count the states faithfully, i.e. each distinct wave function should appear exactly once.

If the single-particle wave functions are separable in Cartesian coordinates, there is a natural organizing principle for counting all states. Each many-body Slater determinant built out of such single-particle wave functions has some number of single-particle nodes in each direction in space, say $n_{x}, n_{y}, n_{z}$, for $d=3$. The list of all Slater determinants with a given total number of nodes $E \equiv n_{x}+n_{y}+n_{z}$ is evidently finite. Increasing the total 
number of nodes one by one, all possible wave functions appear exactly once, so they can be counted faithfully.

The above scheme introduces the important notion of grading, which is just counting the total number of nodes $E$. All $N$-body wave functions spanned by Slater determinants of the same grade $E$ form a closed subspace of the Hilbert space, because a linear combination of such functions is itself a wave function of the same grade.

Clearly, one realization of this scheme is the familiar harmonic-oscillator well, for which the grade $E$ is also the energy, so that the graded states are simultaneously energy eigenstates, and the sum over states, organized by grade, also has the usual thermodynamic meaning. Although the harmonic-oscillator picture is very useful for the visualization of various results, it should not be construed that they are valid only for the oscillator. Even the limitation to separable single-particle wave functions is not strictly necessary. It is retained throughout this article to fix ideas, because it easily produces explicit formulas. The main result is an intrinsic property of $N$ body Hilbert space, because the dimension of an algebra (the number of its generators) does not depend on any particular realization.

In order to implement the main idea from the Introduction, one should see how many $N$-body wave functions in $d$ dimensions one can obtain which are separable across the space dimensions. If the $N$-body functions in one dimension are counted by some partition function, call it $Z_{E}$, then all separable states are counted by $\left(Z_{E}\right)^{d}$. These are explicitly constructed by labeling the space directions with different colors, and combining the corresponding $1 \mathrm{D}$ wave functions in all possible ways. It follows from this interpretation that these states are not all that can be found when $d>1$. The reason is that the Pauli principle operates only upon the exchange of the full (vector) coordinates of a pair of particles, while the iterated $d=1$ states impose the antisymmetrization for each axis (coordinate projection) individually, so that they are too restrictive when $d>1$.

Therefore the essential idea of the present classification is to write the partition function of the $d$-dimensional system of $N$ identical fermions as

$$
Z_{d}=\left(Z_{E}\right)^{d} P_{d}(N, q)
$$

For $d=1$ the ansatz reduces to

$$
Z_{1}=Z_{E} P_{1}(N, q) \equiv Z_{E} q^{E_{g s}(N)}, \quad q=e^{-\beta \epsilon},
$$

where $E_{g s}(N)$ is the ground-state energy in units of $\epsilon$. The "extra" states allowed by the Pauli principle for $d>1$ are counted by the factor $P_{d}$, which reduces in one dimension to a single monomial, the "energy shift" which counts the nodes of the ground state wave function. These "extra" states are called shapes, and the term $P_{d}$ which counts them turns out to be a polynomial for $d>1$, called the shape polynomial. For a graded counting scheme (harmonic well), $Z_{E}$ was first obtained by Euler [5, hence the index $E$. $\left(Z_{E}\right.$ is the same for bosons and fermions, only $E_{g s}$ is different [5, 10.) It will be shown now that the total number of shapes is finite, $P_{d}(N, q=1)=N !^{d-1}$, independently of any particular counting scheme, which proves that $P_{d}$ as defined above is always a polynomial.

\section{B. High-temperature limit}

The non-interacting partition function for $N$ fermions in $d$ dimensions obeys the well-known recursion relation 11, 12

$$
Z_{d}(N, \beta)=\frac{1}{N} \sum_{m=1}^{N}(-1)^{m+1} z_{d}(m \beta) Z_{d}(N-m, \beta) .
$$

Here $z_{d}(\beta) \equiv Z_{d}(1, \beta)$ is the one-particle partition function, while $Z_{d}(0, \beta) \equiv 1$. In the infinite-temperature limit $\beta \rightarrow 0$, or $q \rightarrow 1$, the term $m=1$ dominates the sum on the right, because the factor $z_{d}(m \beta)$ is then the same for all values of $m$, while the factor $Z_{d}(N-m, \beta)$ for $m=1$ strongly dominates those with $N-2$ and less particles, when the temperature is high. Inserting the ansatz (2), one gets $\left(Z_{E}=Z_{1}\right.$ at $\left.q=1\right)$

$$
\begin{aligned}
& N Z_{1}(N, 0)^{d} P_{d}(N, 1) \\
& \quad=z_{d}(0) Z_{1}(N-1,0)^{d} P_{d}(N-1,1) .
\end{aligned}
$$

At $\beta=0, Z_{1}(N, 0)=z_{1}(0)^{N} / N$ ! (classical limit with Boltzmann counting), so that

$$
\begin{aligned}
& N z_{1}(0)^{N d} P_{d}(N, 1) / N !^{d} \\
& \quad=z_{d}(0) z_{1}(0)^{d(N-1)} P_{d}(N-1,1) /(N-1) !^{d} .
\end{aligned}
$$

Because the kinetic energy is additive in the space dimensions, we have $z_{d}(0)=z_{1}(0)^{d}$, so that finally

$$
P_{d}(N, 1)=P_{d}(N-1,1) N^{d-1},
$$

which gives

$$
P_{d}(N, 1)=N !^{d-1},
$$

as advertised in the Introduction. This result is general and exact, because any system is a gas at sufficiently high temperature. Taking logarithms, it means that the non-trivial states (shapes) have an extensive but finite contribution to the free energy, which saturates at sufficiently high temperature. Because the number of shapes is finite, $P_{d}$ is a polynomial.

\section{The shape polynomial}

The above asymptotic result has been obtained without reference to any particular counting scheme, or even one-body separability: there are always $N !^{d-1}$ manybody wave functions which cannot be induced from 
the one-dimensional ones. Among all general counting schemes, the grading scheme is distinguished by the partition function being explicitly solvable. In one dimension, the sum over fermion states as counted by nodes is 10 .

$$
\begin{aligned}
Z_{1}= & \sum_{0 \leq n_{1}<\ldots<n_{N}<\infty} q^{n_{1}+\ldots+n_{N}} \\
& =q^{N(N-1) / 2} \frac{1}{1-q} \cdot \frac{1}{1-q^{2}} \cdots \frac{1}{1-q^{N}}
\end{aligned}
$$

a result due to Euler [5]. (In the harmonic oscillator interpretation, this result counts the states of $N$ fermions in a $1 \mathrm{D}$ oscillator well, with $\varepsilon_{n}=n \hbar \omega$ and $q=e^{-\beta \hbar \omega}$.)

Comparing Eqs. (3) and (9) identifies the 1D partition function $Z_{E}$ [5]. Hence the ansatz (2) for the $d$ dimensional partition function reads

$$
Z_{d}(N, \beta)=P_{d}(N, q)\left(\prod_{k=1}^{N} \frac{1}{1-q^{k}}\right)^{d}
$$

The $d$-th power gives the trivial extension of the 1D case to $d$ dimensions. The extra term $P_{d}(N, q)$ can now be calculated explicitly, proving that the ansatz is solvable when $Z_{E}$ counts wave functions by grade.

Inserting the ansatz (10) into the recursion (4) gives (this formula was first derived by D. Svrtan)

$$
N P_{d}(N, q)=\sum_{k=1}^{N}(-1)^{k+1}\left[C_{k}^{N}(q)\right]^{d} P_{d}(N-k, q),
$$

where $P_{d}(0, q)=P_{d}(1, q)=1$, and

$$
C_{k}^{N}(q)=\frac{\left(1-q^{N}\right) \cdots\left(1-q^{N-k+1}\right)}{\left(1-q^{k}\right)}
$$

is a polynomial, because $k$ always divides one of the $k$ successive powers of $q$ in the numerator. Therefore $P_{d}(N, q)$ is also a polynomial, as expected for a generating function of a finite number of states. For the oscillator, the degree of this polynomial is the energy cutoff above which the shapes saturate.

Significantly, the recursion (11) includes the groundstate shift $E_{0}$ into the degree of the polynomial $P_{d}(N, q)$, which provides formal reason to claim that the ground state is a shape. This carries over even to $d=1$, where $P_{d}$ consists of a single term. The recursion finds the ground-state number of nodes ("energy") as the lowest power of the polynomial $P_{d}(q)$.

\section{BOSONISATION OF THE 1D FERMI GAS}

\section{A. Euler bosons}

Apart from the finite shift ("ground-state energy") $N(N-1) / 2$, the remaining terms in Eq. (9) may be interpreted as the appearance of $N$ harmonic oscillators, mutually distinguishable, each having a different energy spacing, $\hbar \omega_{k}=k \hbar \omega, k=1, \ldots, N$, but without a zeropoint energy of their own. The principal purpose of this section is to obtain the wave functions of these Euler bosons.

The factored form of Eq. (9) suggests that an arbitrary excited state consists of two independent parts, so that its wave function may be factored as well,

$$
\Psi \equiv \Phi \Psi_{0},
$$

where $\Psi_{0}$ is the ground-state Slater determinant, and $\Psi$ an arbitrary one, describing an excitation in terms of some single-particle functions $\phi_{k}(x)$. Here $\Psi_{0}$ accounts for the ground-state shift, while $\Phi$ is a symmetric function in the $N$ variables, defined above as the ratio of the two Slater determinants. The principal observation now is that any $1 \mathrm{D}$ Slater determinant $\Psi$ is divisible by the ground-state determinant $\Psi_{0}$, therefore $\Phi$ is a concrete symmetric polynomial, so that Eq. (13) is not just a notational trick. Namely, the 1D single-particle wave functions consist of three parts,

$$
\phi_{k}(x)=N_{k} p_{k}(x) g(x),
$$

a norm $N_{k}$ which depends only on the quantum number, but not on the variable, an orthogonal polynomial $p_{k}(x)$ which depends on both, and possibly a localization (e.g. exponential) term $g(x)$ which depends on the variable but not on the quantum numbers. The norm and localization terms can be factored out from the Slater determinants, because these have the same quantum numbers in each row, and the same variable in each column. These terms cross out in the numerator and denominator, up to a trivial overall factor. Therefore the only parts remaining in the determinants themselves are the orthogonal polynomials.

A Slater determinant of polynomials is itself a polynomial. It vanishes whenever any two variables are equal, $x_{i}=x_{j}$ for $i \neq j$. By the fundamental theorem of algebra, it must contain a term $\left(x_{i}-x_{j}\right)$ in its root factorization for all pairs $i \neq j$. The denominator $\Psi_{0}$ contains all these terms to lowest order, because the ground state has the smallest number of nodes. Hence it divides the numerator $\Psi$. [The same conclusion applies when $p_{k}(x)$ are trigonometric functions, which are algebraically just shifted polynomials, $\cos k x \leftrightarrow u^{k}+u^{-k}$.]

The above reasoning is reduced to its essence if each single-particle wave function is replaced by a symbolic power counting the number of nodes,

$$
\phi_{k}\left(x_{i}\right) \rightarrow t_{i}^{k}, \quad i=1, \ldots, N .
$$

In this form it appears in mathematics textbooks, which leave the "general" polynomial case as an exercise, for the reader to be convinced that it brings nothing new [13. The denominator $\Psi_{0}$ then becomes the well-known Vandermonde determinant [13,

$$
\Psi_{0}=\Delta\left(t_{1}, \ldots, t_{N}\right) \equiv \prod_{1 \leq i<j \leq N}\left(t_{i}-t_{j}\right) .
$$


This symbolic-power representation is the level of abstraction which we adopt now. One can always specialize to the single-particle wave functions for a particular problem by a reverse of the same mapping, the important point being that it preserves the grading. The scheme works because it encodes the essential behavior of nodes under multiplication and addition of functions. If two functions are multiplied, the number of nodes is added. If the functions are added, the number of nodes stays the same as that of the function with the larger number of nodes. Pure powers behave in exactly the same way. In this abstract representation, the ratio $\Phi$ is called the Schur function 14] (see the Appendix). The physical statement that the Slater determinants span the whole Hilbert space is mirrored by the statement that the Schur functions are a complete basis for the symmetric polynomials.

From a physical point of view, the new insight is that there is "really" only one antisymmetric many-body function in one dimension, and that is the ground-state Slater determinant:

$$
\sum_{i} c_{i} \Psi_{i}=\left(\sum_{i} c_{i} \Phi_{i}\right) \Psi_{0}
$$

where $\Psi_{i}$ are arbitrary Slater determinants, and $\Phi_{i}$ are the corresponding Schur functions. Clearly the term in parentheses is a bosonic wave function. The factored form reflects the factored sum over states $(9)$, where $\Psi_{0}$ accounts for the constant term $q^{N(N-1) / 2}$, corresponding to the ground-state. The remaining question is, which wave functions correspond to the geometric series in the other factor of the partition function. These are the wave functions of the Euler bosons, which describe all possible excitations.

One can guess the correct abstract form by considering the harmonic oscillator, because for the latter there exists an explicit realization of the mapping $(15)$. It is the Bargmann transform 15, 16, which carries Hermite functions $\psi_{n}(x)$ of a real variable $x$ into powers of a complex variable $t$ :

$$
\mathcal{B}\left[\psi_{n}\right](t)=\frac{1}{\pi^{1 / 4}} \int_{\mathbb{R}} e^{-\frac{t^{2}+x^{2}}{2}+x t \sqrt{2}} \psi_{n}(x)=\frac{t^{n}}{\sqrt{n !}} .
$$

The Bargmann-transformed oscillator Hamiltonian is then

$$
H=\sum_{i=1}^{N}\left(t_{i} \partial_{t_{i}}+1 / 2\right) \hbar \omega .
$$

Inserting the decomposition (13) into the Schrödinger equation $H \Psi=E \Psi$, the equation for $\Phi$ becomes

$$
\sum_{i=1}^{N}\left(t_{i} \partial_{t_{i}}\right) \Phi=\frac{E-E_{0}}{\hbar \omega} \Phi,
$$

which is clearly solved by any homogeneous polynomial in the $t_{i}$. Notice how the zero-point term from $\hbar \omega / 2$ has been absorbed into $E_{0}$, i.e. the left-hand side lacks the usual $1 / 2$. This equation is "first-quantized," because the requirement that $\Phi$ be symmetric in the $t_{i}$ must be added extraneously. To obtain a primitive realization of the symmetry requirement ("second quantization"), invoke the change of variables

$$
\begin{aligned}
& e_{1}=t_{1}+\ldots+t_{N}=\sum_{i} t_{i} \\
& e_{2}=t_{1} t_{2}+t_{1} t_{3}+\ldots+t_{N-1} t_{N}=\sum_{i<j} t_{i} t_{j}, \\
& \quad \vdots \\
& e_{N}=t_{1} t_{2} \cdots t_{N} .
\end{aligned}
$$

The $e_{k}$ are just the elementary symmetric functions, appearing e.g. in the Viète formulas for the coefficients of the polynomial $\left(t-t_{1}\right) \cdots\left(t-t_{N}\right)$, whose roots are the $t_{i}$. As is well known, the transformation from roots to coefficients is regular as long as all $t_{i} \neq t_{j}$. Its Jacobian in Bargmann space (volume element $d \operatorname{Re} t_{i} d \operatorname{Im} t_{i}$ ) is the square of the Vandermonde determinant (16), $J=|\Delta|^{2}$.

All symmetric functions in the $t_{i}$ can be rewritten in the $e_{k}$. The $e_{k}$ are eigenfunctions of Eq. 20 with eigenvalue $k$, so the Hamiltonian is transformed to the $e_{k}$ basis as

$$
\sum_{i=1}^{N}\left(t_{i} \partial_{t_{i}}\right)=\sum_{k=1}^{N} k\left(e_{k} \partial_{e_{k}}\right)
$$

whose eigenfunctions are all the monomials $e_{1}^{n_{1}} \cdots e_{N}^{n_{N}}$, with eigenvalue $n_{1}+2 n_{2}+\ldots+N n_{N}$, and no symmetry restrictions: the $e_{i}$ are therefore distinguishable, as implied by Euler's factorization in Eq. (9). Hence functions of the $e_{k}$ are a second-quantized representation for the original many-body fermionic excitations, yet the representation is purely bosonic. The $e_{k}$ are a complete basis for the symmetric functions, and all their monomials are generated by the formal expression

$$
\frac{1}{1-e_{1}} \cdot \frac{1}{1-e_{2}} \cdots \frac{1}{1-e_{N}} \text {. }
$$

Because $e_{k}$ has the eigenvalue $k$, substituting $e_{k}=q^{k}$ in the above expression will give the corresponding canonical partition function, recovering Euler's result. Obviously, the monomial $e_{1}^{n_{1}} e_{2}^{n_{2}} \ldots e_{N}^{n_{N}}$ is the wave function of $n_{1}$ Euler bosons of type $1, n_{2}$ of type 2, etc. This identification is the main result of the present section. It obviously carries over to the formal-power representation, again because the energy in the oscillator case is the grading, or polynomial degree, in the general case.

Two things have been accomplished by identifying the Euler bosons. The most important one is finding a generating function for their wave functions, Eq. (23), which corresponds precisely to the sum over states which counts them, Eq. (9). This will enable "lifting" the present result to $d$ dimensions by way of Eq. 10 and thus identifying the wave functions of the shapes, counted by $P_{d}(N, q)$, which is the main purpose of the present article. 
The other is a more qualitative development: Euler boson excitations have direct physical connotations. Namely, the transformation (21) is non-linear, progressing from a pure sum to a pure product. In physics, product wave functions correspond to (non-interacting) gases, while sum wave functions are typically used as trial wave functions for liquids. In other words, the progression from $e_{1}$ to $e_{N}$ is physically in terms of decreasing collectivity: the lowest-grade Euler boson $e_{1}$ is the most collective (liquid-like), while the highest-grade $e_{N}$ is least collective (most gas-like). This simplicity of physical interpretation pleasantly reflects their mathematical simplicity, because of which they may be readily calculated by Viète's interpretation above, deserving the name elementary symmetric functions. By contrast, Schur functions are sophisticated combinatorial objects. The most efficient prescription for their calculation is to interpret them as generating functions of semi-standard Young tableaux, which is quite a surprising insight [14 (see the Appendix). There is no simple physical interpretation of this property, accounting perhaps for the fact that representations of collective states in terms of Slater determinants are rarely physically transparent.

\section{B. Deflation algorithm}

In the previous section, it was found that Euler bosons are the natural basis of graded one-dimensional $N$-fermion wave functions. Slater determinants are in a sense redundant: only one Slater determinant, the ground state, is sufficient to generate the whole Hilbert space, with excitations described in terms of Euler bosons. In order to generalize this result to more than one dimension, it is necessary to obtain the Euler-boson wave functions explicitly, in terms of standard Slater determinants.

First one must deal with a slight complication. Compare the wave functions $e_{2} \Psi_{0}$ and $e_{1}^{2} \Psi_{0}$, say. Because $e_{1}^{2}=\left(t_{1}+t_{2}+\ldots\right)^{2}$ contains terms like $t_{1} t_{2}$, which also appear in $e_{2}=t_{1} t_{2}+\ldots$, the two wave functions will not be orthogonal. It is much better to interpret the powers $e_{i}^{k}$ appearing in Eq. (23) by raising individual monomials in them to the required power without cross terms, e.g. $e_{1}^{2} \rightarrow t_{1}^{2}+t_{2}^{2}+\ldots$, or in general:

$$
e_{m}^{k} \rightarrow \sum_{1 \leq i_{1}<\ldots<i_{m} \leq N}\left(t_{i_{1}} \cdots t_{i_{m}}\right)^{k}
$$

which clearly keeps the terms orthogonal, because now no monomial appears twice in the various geometric series. [Technically Eq. 24) is a composition, or plethysm, of the $e_{m}$ and power sums $p_{k}$ [14.]

The deflation algorithm operates as follows. Take any monomial wave function containing Euler bosons, e.g. $e_{1} e_{2}^{2} \Psi_{0}$. By power counting, this state belongs to the fifth-excited "oscillator" level above the ground state. Expand it as a polynomial in the formal variables $t_{i}$. All
Slater determinants in the fifth level can be similarly expressed as polynomials in the same $t_{i}$. Now it is simply a matter of ordering the polynomial terms in some definite (say lexicographic) order, to see which Slater determinant contains the leading order monomial of the given polynomial wave function, and subtracting it with the appropriate coefficient. Then the leading power of the remainder is determined, and subtracted in the same way. Because the Slater determinants are a complete orthogonal basis for each level, this procedure is guaranteed to terminate.

In fact the procedure is redundant. The Slater determinants can themselves be factored as in Eq. (13), so there is no need to multiply out the term $\Psi_{0}$. The problem boils down to expressing a given product of sums like (24) in Schur functions, which is just a basis transformation among symmetric functions. The reason for stating the algorithm in the less efficient formulation is that it then generalizes directly to several dimensions, where the analogous generalization of Schur functions is not available.

It is essential for the deflation algorithm that one deal with Slater determinants of unnormalized single-particle states. In practice, this means using formal powers $t_{i}^{k}$, instead of $t_{i}^{k} / \sqrt{k !}$ as in Bargmann space. All superpositions of Slater determinants are obtained among such unnormalized determinants, and normalized as superpositions only after being mapped back to some concrete realization. This will become clear in the example in the second part of the paper.

The above technical considerations reflect a change of viewpoint. The deflation algorithm in the algebraic approach corresponds to taking projections in the standard functional-analytic approach. The algebraic approach, chosen by the mapping (15), brings one to consider $N$ fermion Hilbert space as a space of antisymmetric polynomials, graded by their degree. In one dimension, this space maps straightforwardly on the space of symmetric polynomials, which is one way to understand why the fermion sign problem [17 is trivial when $d=1$. While this insight is undoubtedly interesting, the true advantage of the algebraic approach appears in more than one dimension. There it uncovers a fundamental structure of many-body Hilbert space which is hidden in the functional-analytic approach, as will become apparent in Sect. IVB below.

\section{THE MULTIDIMENSIONAL CASE}

\section{A. Slater determinants}

A Slater-determinant state is obtained by denoting single-particle wave functions as formal powers in $d$-plets of variables for each particle, say the triplet $t, u, v$ for $d=3$. Then a general (unnormalized) Slater determi- 
nant is written e.g. for $N=2$ particles

$$
\begin{aligned}
\left|\vec{m}_{1}, \vec{m}_{2}\right|=\left|t_{1}^{m_{11}} u_{1}^{m_{12}} v_{1}^{m_{13}}, t_{2}^{m_{21}} u_{2}^{m_{22}} v_{2}^{m_{23}}\right| \\
=\left|\begin{array}{lll}
t_{1}^{m_{11}} u_{1}^{m_{12}} v_{1}^{m_{13}} & t_{2}^{m_{11}} u_{2}^{m_{12}} v_{2}^{m_{13}} \\
t_{1}^{m_{21}} u_{1}^{m_{22}} v_{1}^{m_{23}} & t_{2}^{m_{21}} u_{2}^{m_{22}} v_{2}^{m_{23}}
\end{array}\right| .
\end{aligned}
$$

An absolute ordering (e.g. lexicographic) on the $d$ dimensional integer vectors $\vec{m}_{i}, i=1, \ldots, N$, is required to fix the phase of the Slater determinants, which need not be explicit here. An example, to be used later, is the ground state of $N=3$ particles in $d=2$ dimensions:

$$
g_{0} \equiv|(1,0),(0,1),(0,0)|=\left|t_{1}, u_{2}, 1\right|=\left|\begin{array}{ccc}
t_{1} & t_{2} & t_{3} \\
u_{1} & u_{2} & u_{3} \\
1 & 1 & 1
\end{array}\right| .
$$

\section{B. General algorithm for shape wave functions}

As noted before, the factorized form of the wave function (17) follows the factorization (9) of the sum over states. Similarly the $d$-dimensional sum over states 10 implies the general form 4

$$
\sum_{i=1}^{P_{d}(N, q=1)} \Phi_{i} \Psi_{i}
$$

for wave functions, where the $\Phi_{i}$ are $d$-dimensional Euler boson states, and the $\Psi_{i}$ are all the $P_{d}(N, q=1)$ states counted by the factor $P_{d}(N, q)$ in the expression (10). These states have been called shapes above. Clearly they generalize the ground-state Slater determinant in Eq. 17) in such a way that an arbitrary wave function can be expressed in terms of shapes, which must be antisymmetric wave functions, because the $\Phi_{i}$ are symmetric. In Sect. IIB, it has been proven completely generally that $P_{d}(N, q=1)=N !^{d-1}$, therefore the whole $N$-fermion Hilbert space can be finitely generated with the shapes as basic antisymmetric building blocks. They are generators of the finite-dimensional Hilbert-space algebra induced by wave-function multiplication. This is the main result of the present work, announced in the Introduction. The purpose of this section is to generate the shape basis explicitly, amounting to a constructive proof of the same result.

One can combine the deflation algorithm with the $d$ dimensional extension of Eq. 23) to obtain all the shapes. All possible Euler boson wave functions in $d$ dimensions are obtained simply by multiplying $d$ copies of Eq. (23), one for each set of variables $t_{i}, u_{i}, \ldots$, representing the directions in space:

$$
\left[\frac{1}{1-e_{1}(t)} \cdots \frac{1}{1-e_{N}(t)}\right]\left[\frac{1}{1-e_{1}(u)} \cdots \frac{1}{1-e_{N}(u)}\right] \cdots
$$

where e.g. $e_{1}(u)=u_{1}+\ldots+u_{N}$, and so on. There is no similar closed generating formula for the wave functions of the shapes, which would analogously correspond to the shape-polynomial factor $P_{d}(N, q)$ in the counting expression 10 . Instead we resort to the following constructive algorithm.

Start with shapes at zeroth level, which are just the Slater determinants spanning the (possibly degenerate) ground state level, which contains no Euler bosons. Excite them by multiplying them with Euler bosons, noting that there are only $d$ Euler bosons which carry one quantum of excitation, namely the $e_{1}$ monomials, one for each direction in space. Multiplying the ground state(s) with them gives all the states containing Euler bosons ("trivial states" for short) at the first excited level, so if it contains more than $d$ states, the remainder (orthogonal complement) are the shapes at first level. After applying the deflation algorithm to find the span of the trivial states in terms of Slater determinants, a standard algebraic algorithm is invoked to find the orthogonal complement of this vector space. The dimension of the complement space is given by the corresponding coefficient in the shape polynomial $P_{d}(N, q)$, which is a useful check on the implementation. Now one iterates the procedure, multiplying the first-level shapes with the Euler bosons $e_{1}$, and adding the ground states multiplied by all twoquanta bosons, like $e_{2}$ and $e_{1}^{2}$, to obtain all the trivial states in the second level. The span of the so-generated second-level trivial space is again found by the deflation algorithm. The second-level shapes are the orthogonal complement to that space, and so on until all shapes predicted by the shape polynomial are found. In this way, the algorithm finds the Hilbert space span of the shapes explicitly, defining them rigorously up to basis transformations in the orthogonal-complement space at each level. The constructive proof of Eq. 27) is thus complete.

The algorithm is not efficient, because it finds all the states, while the number of trivial states rises quickly even as the shapes die out. E.g. for $N=3$ particles in $d=3$ dimensions, the total number of shapes is $3 !^{2}=36$. The shape polynomial reads

$$
P_{3}(3, q)=q^{9}+3 q^{7}+7 q^{6}+6 q^{5}+6 q^{4}+10 q^{3}+3 q^{2}
$$

(note the triply degenerate ground state), so there is a single shape in the seventh excited level (coefficient of $\left.q^{9}\right)$. But the degeneracy of the seventh level is 3838 , so the algorithm spends most of its time finding the span of the 3837 trivial states, in order to extract the last single shape.

The $d$-th power appearing in the factorization 10 , as reflected by Eq. 28 in the above construction, allows a refinement of the expression 27) in general. Namely, the terms $\Phi_{i}$ can always be written, e.g. in three dimensions,

$$
\Phi_{i}=\sum_{j k l} c_{j k l}^{i} \Phi_{i j}^{x} \Phi_{i k}^{y} \Phi_{i l}^{z},
$$

where the $\Phi^{x, y, z}$ are Euler-boson monomials, each corresponding to a particular direction in space (Cartesian 
axis). In other words, the $\Phi_{i}$ are superpositions of terms independently symmetric in the $N$ variables (coordinate components) along each of the $d$ directions in space. This form is far from the most general one in all $N d$ coordinates, symmetric upon exchange of any two particle indices. [For example, terms like $t_{1} u_{2}+t_{2} u_{1}$ cannot appear alone, but only embedded in factored expressions like $\left(t_{1}+t_{2}\right)\left(u_{1}+u_{2}\right)$.] It is interesting that such a strong restriction on the coefficients $\Phi_{i}$ still generates the whole $N$-fermion Hilbert space. Physically, it means that the shapes are the only "genuinely" $d$-dimensional states; all excitations of the shapes may be reached as if the directions in space were different colors.

On a lesser note, the trivial states generated by the algorithm are not always orthogonal, because multiplication of various shapes with different Euler bosons can generate the same monomials. Experience with standard quantum chemical calculations [18 suggests that little would be gained by orthogonalizing these vectors explicitly, especially because the overlap matrices among the trivial states are quite sparse. There is a physical interpretation both of the overlaps, and of the sparseness. The overlap indicates the possibility that exciting some shape with an Euler boson, and then shedding a different Euler boson, will give another shape. Such reconfiguration by excitation is observed sometimes, but cannot be too easy if the shapes are robust, hence the sparseness.

\section{EXAMPLES}

\section{A. The case $d=2$ and $N=3$}

The present example serves as an illustration of the algorithm, and of the inverse mapping which recovers a concrete realization of the shapes from the abstract representation. It is the simplest non-trivial multidimensional case. The partition function 10 is

$$
\begin{aligned}
& \left(q^{2}+4 q^{3}+q^{4}\right)\left[\left(\frac{1}{1-q}\right)\left(\frac{1}{1-q^{2}}\right)\left(\frac{1}{1-q^{3}}\right)\right]^{2} \\
& =q^{2}+(1 \cdot 2+4) q^{3}+(1 \cdot 5+4 \cdot 2+1) q^{4}+\ldots,
\end{aligned}
$$

where $q^{2}+4 q^{3}+q^{4}=P_{2}(3, q)$ is the shape polynomial, predicting six shapes, one of which is the ground state, Eq. 26. The first-excited manifold is spanned by six Slater determinants:

$$
\begin{aligned}
& \left|t_{1}^{2}, t_{2}, 1\right| \equiv g_{11}, \quad\left|t_{1} u_{1}, t_{2}, 1\right| \equiv g_{12} \\
& \left|u_{1}^{2}, t_{2}, 1\right| \equiv g_{13}, \quad\left|t_{1}^{2}, u_{2}, 1\right| \equiv g_{14} \\
& \left|t_{1} u_{1}, u_{2}, 1\right| \equiv g_{15}, \quad\left|u_{1}^{2}, u_{2}, 1\right| \equiv g_{16} .
\end{aligned}
$$

The deflation algorithm gives the Euler-boson states at first level:

$$
\begin{aligned}
& e_{1}(t) g_{0}=\left(t_{1}+t_{2}+t_{3}\right) g_{0}=-g_{12}+g_{14}, \\
& e_{1}(u) g_{0}=\left(u_{1}+u_{2}+u_{3}\right) g_{0}=-g_{13}+g_{15},
\end{aligned}
$$

where $g_{0}$ is the ground state $(26)$. The four states orthogonal to them are the shapes predicted by the term $4 q^{3}$ in $P_{2}(3, q)$ :

$$
\begin{aligned}
& S_{11}=g_{11}, \quad S_{12}=g_{12}+g_{14}, \\
& S_{13}=g_{13}+g_{15}, \quad S_{14}=g_{16} .
\end{aligned}
$$

At the second level, spanned by 14 Slater determinants, the partition function breaks down the multiplicity as $14=1 \cdot 5+4 \cdot 2+1$, which amounts to: (a) the groundstate $g_{0}$ multiplied by any of $e_{1}(t)^{2}, e_{1}(u)^{2}, e_{1}(t) e_{1}(u)$, $e_{2}(t)$, or $e_{2}(u)$; (b) any of the four shapes (34) at first level, multiplied by either $e_{1}(t)$ or $e_{1}(u)$; (c) finally the last shape, orthogonal to the 13 trivial states just listed. It is

$$
\begin{aligned}
S_{2}=\left|t_{1} u_{1}^{2}, t_{2}, 1\right|-\left|t_{1}^{2} u_{1}, u_{2}, 1\right| \\
+\left|t_{1}^{2}, u_{2}^{2}, 1\right|-\left|t_{1} u_{1}, t_{2}, u_{3}\right| .
\end{aligned}
$$

To visualize these states in real space, one must map the abstract (node-counting) representation back to some concrete realization. A standard model for electrons in a quantum dot is to place them in a harmonic oscillator potential [7, 8]. For the oscillator potential, the required inverse of the mapping $[15$ is

$$
t_{i}^{k} \rightarrow \phi_{k}\left(x_{i}\right)=H_{k}\left(x_{i}\right) e^{-x_{i}^{2} / 2},
$$

and similarly for the other directions, with $H_{k}$ the Hermite polynomial. This mapping operates uniquely only on monomials like $t^{k} u^{m}$, because $t^{k} t^{m}=t^{k+m}$ does not imply $\phi_{k}(x) \phi_{m}(x)=\phi_{k+m}(x)$. Hence it should be applied to factored expressions like $\Phi \Psi$ only after expanding them in the abstract representation first.

The normalized single-particle densities corresponding to $S_{11}, S_{12}$, and $S_{2}$ are shown in Fig. 1. Note that $S_{11}$ is just the ground state of the one-dimensional system, appearing as a first-excited state in two dimensions. $S_{13}$ and $S_{14}$ are rotated by $90^{\circ}$ with respect to $S_{11}$ and $S_{12}$, so there are only four "essentially" different shapes, not six, including the ground state. Obviously, this redundancy is related to the invariance under relabeling of the axes.

Notably, the shape $S_{12}=g_{12}+g_{14}$ and the trivial state $e_{1}(t) g_{0}=-g_{12}+g_{14}$ have the same single-particle density matrix, because the Slater determinants $g_{12}$ and $g_{14}$ differ in two orbitals, so the cross-terms $g_{12} \cdot g_{14}$ vanish when integrated in all but one variable. This means they are part of the same manifold of wave functions over which the density functional is determined by minimization in the Hohenberg-Kohn [19] approach, for a given density $n$ :

$$
F[n]=\min _{\{\Psi: \rho[\Psi]=n\}}\left\langle\Psi\left|\hat{H}_{0}+\hat{V}_{e e}\right| \Psi\right\rangle .
$$

They differ in the correlation (two-particle) density matrix, as shown in Fig. 2,

The whole discussion above could have been carried out equally well for electrons in a box, with the mapping

$$
t_{i}^{k} \rightarrow \cos k x_{i}, \quad u_{i}^{k} \rightarrow \cos k y_{i}
$$



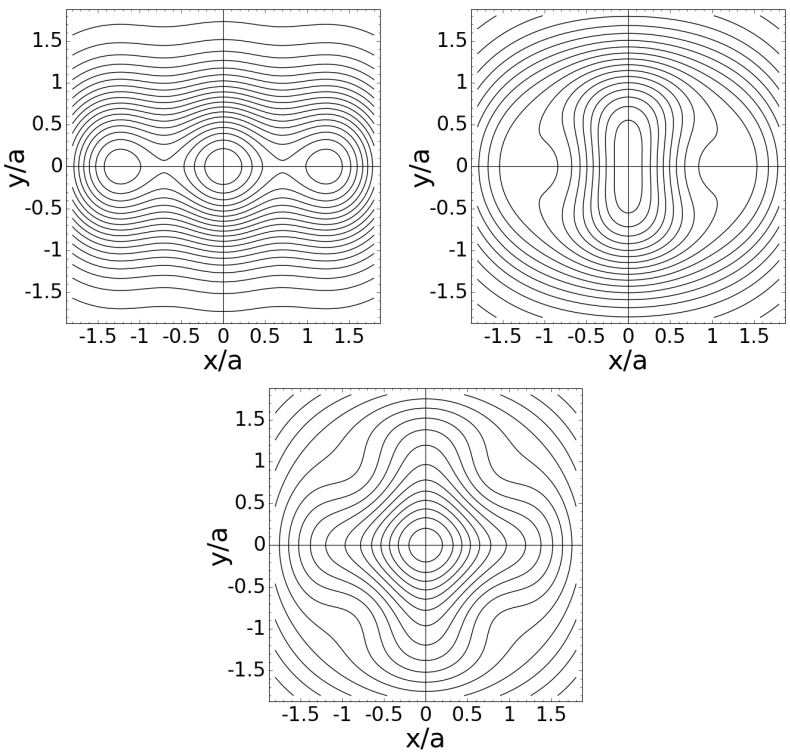

FIG. 1. Single-particle densities for $S_{11}, S_{12}$, and $S_{2}$ in the oscillator potential. The coordinate scale is the oscillator length $a=\sqrt{\hbar / m \omega}$.
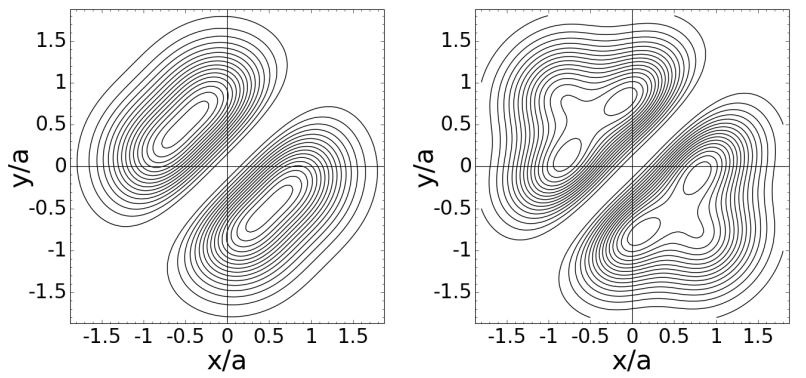

FIG. 2. Two-particle densities for $e_{1}(t) g_{0}$ and $S_{12}$ in the oscillator potential, along the cut $\vec{x}_{1}=(x, x)$ and $\vec{x}_{2}=(y, y)$. The scale is the same as in Fig. 1

for open boundary conditions, replacing $\cos k x, y$ with $\sin (k+1) x, y$ for closed boundary conditions. In any realization, the six shapes span the whole space of antisymmetric three-body states in two dimensions, using only symmetric-function coefficients.

\section{B. Coulomb interaction}

For the repulsive Coulomb interaction between fermions in a harmonic well, small numerical experiments in $d=2$ and $d=3$ invariably favor the shapes as giving a smaller value of the Coulomb repulsion $\left\langle\Psi\left|\hat{V}_{e e}\right| \Psi\right\rangle$, over the trivial basis states of the form $\Psi_{T}=\Phi \Psi_{1}$, where $\Psi_{1}$ is some shape, excited by a symmetric term $\Phi \neq 1$, taken nodeless (otherwise anything can be construed). Plausibly, $\Phi$ seems to act as a coherent amplification for the final value of the integral, so that $\Phi=1$ gives a smaller integral overall, which is preferable when the force is repulsive.

Typically, one observes in the numerical experiments that the diagonal Coulomb matrix elements separate a shape clearly from the multiplet of trivial states, spanned by the same Slater determinants. E.g., the state (35), spanned by four vectors, is separated from the remaining triplet. The off-diagonal elements also show the expected pattern, in that they are much smaller among different shapes, than within such multiplets. In other words, it is much more difficult for the Coulomb force to change a shape, than to relax a shape over its related multiplet of trivial states.

The observed effects of the Coulomb force conform to the idea that excited states are organized into bands, such that the lowest state in each band is dominated by a single shape. Such excitation patterns are ubiquitous in finite systems, including nuclei, molecules, and quantum dots, where the lowest state in each band is sometimes called the band-head. Shapes are natural candidates for the band-head states, because, as noted already, they are the only genuinely $d$-dimensional states.

\section{Trial wave functions}

Truncations of the method which builds the whole Hilbert space give rise to specific families of trial wave functions. For example, take two particles in three dimensions. The shape polynomial is $3 q+q^{3}$, and the four shapes are

$$
\begin{aligned}
& \Psi_{1}=t_{1}-t_{2}, \quad \Psi_{2}=u_{1}-u_{2}, \\
& \Psi_{3}=v_{1}-v_{2}, \quad \Psi_{4}=\Psi_{1} \Psi_{2} \Psi_{3} .
\end{aligned}
$$

Contrary to the intuitive idea of shapes in the Introduction, $\Psi_{4}$ does factor over the space dimensions. Such an "accidentally" factored term must appear whenever it, too, gives a possible way to write an antisymmetric function. Here it is the only higher shape, showing that the number of ways an antisymmetric wave function can be constructed is quite restricted for two particles.

A general two-body wave function can be written by combining the $\Psi_{i}$ with symmetric-polynomial coefficients:

$$
\sum_{i=1}^{4} \Phi_{i}(t, u, v) \Psi_{i}
$$

Trial wave functions are obtained by restricting the polynomials in various ways. For example, $\Phi_{i}(t, u, v)=$ $c_{i 0}+c_{i 1} e_{1}(t)+c_{i 2} e_{1}(u)+c_{i 3} e_{1}(v)$. The approach provides a qualitative language to describe the trial functions. Thus, whether $e_{2}$ or $e_{1}^{2}$ is more important in second order is a question with physical meaning, because, as noted before, $e_{2}$ is a gas-like excitation, while $e_{1}^{2}$ is liquid-like. 
The trial wave functions use a relatively small number of Slater determinants, because the shapes themselves are quite sparing in this sense. E.g. $S_{2}$ in Eq. (35) uses only four vectors of the available fourteen. Similarly, the highest shape for $N=3$ in $d=3$ is spanned by 36 Slater determinants out of possible 3838. Finding that particular combination is quite beyond trial and error.

\section{Simulations}

The critical issue is to locate the nodes of many-body functions in the $N d$-dimensional configuration space [20], otherwise the well-known fermion sign problem appears [17. These nodes are unknown, so that simulations use a guiding function whose nodes are supposed to be near those of the exact solution. The shapes provide a finite and complete antisymmetric-function basis for guiding functions in simulations.

The model of spin-polarized electrons confined to a sphere in $d=3$ is of contemporary interest as a test-bed for theory and simulations [21]. Here it means mapping $t, u, v$ to $x, y, z$ and interpreting the latter (Cartesian) coordinates in terms of polar and azimuthal angles on the unit sphere. For two particles, the shapes 39 define the nodal surfaces

$$
x_{1}=x_{2}, \quad y_{1}=y_{2}, \quad z_{1}=z_{2} .
$$

In the space of particle 1 , these are three circles which cut off a cap of the sphere at the coordinates $x_{2}, y_{2}, z_{2}$, respectively, of particle 2. If the Hamiltonian is invariant under coordinate permutations, then $\left\langle\Psi_{i}|H| \Psi_{j}\right\rangle=0$ for $i \neq j, i, j=1,2,3$, so one can choose any one of them for the guiding function without loss of generality, say $\Psi_{3}=z_{1}-z_{2}$. Then the most general ground-state wave function 40 up to relabeling the axes is

$$
\left[\Phi_{1}+\left(x_{1}-x_{2}\right)\left(y_{1}-y_{2}\right) \Phi_{2}\right]\left(z_{1}-z_{2}\right) \equiv\left(z_{1}-z_{2}\right) \widetilde{\Phi},
$$

including $\Psi_{4}$. It follows that the interacting ground state has the same nodes $z_{1}=z_{2}$ as the non-interacting ground state $\widetilde{\Phi}=$ const., assuming $[20,22$ that $\widetilde{\Phi} \neq$ const. does not introduce new nodes. This result was recently derived as a theorem for this particular model [21], while the above reasoning is model-independent, based on the limited number of possible shapes, as listed in Eq. (39).

By the same reasoning, a similar result as 42 can be obtained for the oscillator potential, with the mapping (36). Then the interesting question arises, whether simulating the oscillator in real or complex (Bargmann) space is more convenient, given that complexification doubles the number of real variables. A simulation keeping $z_{1}<z_{2}$ should converge to a form like 42 for a nodeless $\widetilde{\Phi}$, however the natural eigenfunctions of the problem are still the Hermite functions, which can be recovered only by multiplying out the original abstract expression:

$$
\left(v_{1}-v_{2}\right) \widetilde{\Phi} \rightarrow \sum_{\vec{n}} c_{\vec{n}} \Phi_{\vec{n}}(\vec{R}),
$$

where $\Phi_{\vec{n}}(\vec{R})$ contains Hermite functions. The node $v_{1}=v_{2}$ which was explicitly controlled in the Bargmann representation is now hidden under cancellations of oscillating functions. Real-space representations generically have the problem that nodes of the constituent one-body wave functions, required by orthogonality, interfere with the analysis of nodes of the $N$-body function, which are completely different objects [20].

Similar issues arise for standing waves $\cos k x$, which may be avoided by the travelling-wave complexification $e^{i k x}$. As of this writing, it seems that the advantages of having $\phi_{k} \phi_{m}=\phi_{k+m}$ outweigh any disadvantage of complexification. Further considerations along these lines are beyond the scope of this article.

\section{SPACE DIMENSION AND BOSON-FERMION CORRESPONDENCE}

The factorization (2) is the same for identical bosons. The only difference in the recursion for the shape polynomial (11) is that the alternating sign $(-1)^{k+1}$ does not appear, and Slater determinants have to be replaced by permanents (i.e. lose the alternating sign) in the general algorithm. The Euler bosons remain formally the same elementary symmetric function monomials. Indistinguishable (original) bosons are replaced by distinguishable Euler bosons and shapes, in close parallel to the fermion case. This correspondence explains where have "gone" all the most general symmetric functions, alluded to in Sect. IVB above. They span the space of identical bosons, which is however also finitely generated, with coefficients (Euler bosons) as restricted as the ones for fermions. In other words, just as a finite number of antisymmetric $N$-body functions is sufficient to generate them all, so can all symmetric functions be generated from a finite number of genuinely $d$-dimensional bosonic shapes. These symmetric shapes are the only "real" difference between bosons and fermions.

An interesting distinction appears between spaces of odd and even dimension. In even dimensions, shape polynomials are always symmetric. This can be understood by replacing $q \rightarrow 1 / q$ in the recursion (11), which reverses the polynomial. Clearly the net effect on the recursion is that the coefficient $C_{k}^{N}(q)$ gains an extra sign of $(-1)^{k+1}$. Because it is raised to the $d$-th power, this extra sign vanishes in even dimensions, so the recursion for the polynomial and for the reversed polynomial is the same. Therefore the polynomial must be symmetric, in both bosonic and fermionic cases. Such is $q^{2}+4 q^{3}+q^{4}$ in Eq. (31).

The odd-dimensional case is more interesting. Now the sign change $(-1)^{k+1}$ cancels the $(-1)^{k+1}$ in the recursion for the fermionic case, and introduces it in the bosonic case: polynomial reversal changes the bosonic recursion into the fermionic one, and vice versa. This means that the coefficient lists in the shape polynomials for bosons and for fermions are "mirror images" of each other. For 
example, the shape polynomial for $N=3$ bosons in $d=3$ dimensions is

$$
B_{3}(3, q)=1+3 q^{2}+7 q^{3}+6 q^{4}+6 q^{5}+10 q^{6}+3 q^{7},
$$

to be compared with $P_{3}(3, q)$ in Eq. $(29)$.

In odd dimensions, physical inferences can be made between the bosonic case and the fermionic one. For example, every bosonic polynomial $B_{d}(N, q)$ begins with a coefficient of unity, because the bosonic ground state cannot be degenerate - but this statement means that the highest shape in the fermionic polynomial is always non-degenerate. Furthermore, the first excited state for bosons contains no shapes, because its degeneracy is always $d$, and there are also $d$ Euler bosons $e_{1}$, as mentioned before. Therefore, there will be no shapes at the second-highest level for fermions - the absence of the term $q^{8}$ in Eq. 29 mirrors the absence of $q^{1}$ in Eq. (44). For a simple example, $\Psi_{4}$ in Eq. (39) is a secondexcited state, while all nine first-excited states are of the form $\left(a_{1}+a_{2}\right)\left(b_{1}-b_{2}\right)$ with $a, b=t, u, v$.

\section{DISCUSSION}

The main result of this work is a fundamental insight into the structure of $N$-fermion Hilbert space: a finite number of antisymmetric functions generate all antisymmetric functions, with symmetric-function coefficients. This property of being finitely generated does not depend on any particular realization of the Hilbert space [4. It has been made explicit here with the convenient choice of one-body functions separable in Cartesian coordinates. The independence of the main result on such technicalities was demonstrated in the formula (8), which needed only the structural formula (4) to count the shapes directly from the ansatz (2).

Mathematically, Eq. (10) is a Poincaré (a.k.a. Hilbert) series 23, which counts the dimensions of the vector (Hilbert) spaces of a given grade, which may be visualized as the degeneracy of the corresponding oscillator level. The particular form of the series indicates how these spaces may be generated algebraically by combining certain invariant polynomials, called Euler bosons and shapes here. In the standard language of invariant theory [4, the Euler bosons are primary, and the shapes secondary invariants. This identification follows 4 from the most general form of the wave function, e.g. for $d=3$ :

$$
\sum_{i=1}^{N !^{2}} \Phi_{i} \Psi_{i}, \quad \Phi_{i}=\sum_{j k l} c_{j k l}^{i} \Phi_{i j}^{x} \Phi_{i k}^{y} \Phi_{i l}^{z},
$$

where the $\Phi^{x, y, z}$ are monomials of Euler bosons in the three directions, while the $\Psi_{i}$ are all the $N !^{2}$ shapes of $N$ particles in three dimensions. The invariants $\Psi_{i}$ are antisymmetric polynomials over $\mathbb{Z}$ which finitely generate the Hilbert space of $N$ identical fermions as a graded algebra, with coefficients $\Phi_{i}$ from the ring of polynomials over $\mathbb{C}$, independently symmetric in each of $d$ sets of $N$ variables. The grading is by degree of the polynomials, which is just the energy in the oscillator case. Remarkably, but not unexpectedly, the main result (45) is equally valid for bosons and for fermions, with symmetry in place of antisymmetry, and permanents replacing determinants in the constitutive expressions.

Antisymmetric polynomials in one dimension can always be studied by proxy symmetric polynomials: Slater determinants in formal powers and Schur functions differ by a fixed factor, the Vandermonde determinant [14. The present work shows that when $d>1$ antisymmetry gives rise to qualitatively new polynomial invariants, the shapes. They are a different generalization of the Vandermonde determinant than the obvious one, which is just an excited one-dimensional state. This mathematical generalization has a direct physical meaning as the generalization of the Slater-determinant form for the ground state to more than one dimension. The appearance of additional antisymmetric invariants - the shapes - is a consequence of the weakening of the Pauli principle when $d>1$, because it requires antisymmetry only with respect to interchange of vector coordinates, i.e. simultaneous interchange of $d$-plets of variables refering to different particles, as opposed to the interchange of any two variables, which is the case in one dimension.

Particles with different spin projections are distinguishable, so their wave functions can be obtained by a simple direct product of the spaces discussed here. Notably, the shape space is not closed with respect to spin. For $2 N$ spin-up fermions, there are $(2 N) !^{d-1}$ shapes, while for $N$ fermions of spin up and $N$ of spin down, there are only $N !^{2(d-1)}$ shapes, a much smaller number in general. Raising the total spin projection, which makes more particles indistinguishable, increases the choice of shapes, i.e. orbital states with enhanced collectivity. This observation fits well with Hund's rule [24]: spin-polarized states are preferred when Coulomb effects are important.

The direct product of up- and down-spin spaces does not imply that the wave functions have to be in pure product form, which is known to constrain them unphysically [25]. One can assume a configuration-interaction (CI) form, which is the superposition

$$
\sum_{i} c_{i} \Psi_{\uparrow i} \Psi_{\downarrow i}
$$

where the $\Psi_{\sigma i}$ are particular cases of 45. A CI form can describe the topology of the exact nodal surface [25]. By mapping all wave functions onto symbolic polynomials, the algebraic approach puts the discussion of nodalsurface topologies directly into the purview of algebraic geometry, one of whose traditional concerns are the zeros of multivariate polynomials [26, 27. On the other hand, there is always an underlying differentiable manifold, spanned by the original one-body wave functions. For the harmonic oscillator, the Bargmann transform even allows a direct reinterpretation of the same polynomials as analytic functions in complex (Bargmann) space. Physical intuition suggests that possible nodal- 
surface topologies should not depend qualitatively on the confining potential, as long as one can be adiabatically transformed into another. Hence the harmonic-oscillator setting is already quite general, as far as the topology of nodal surfaces is concerned.

Restoration of rotational invariance similarly proceeds by superposition. As already noted, such basis issues cannot impinge on the underlying property of Hilbert space, that it is finitely generated. However, a large part of practical invariant theory [4] is to find optimal sets of generators for particular applications, and the flexibility of the algebraic structure in the choice of generators bodes well for future physics applications. In the present work, an explicit realization of generators organized by grade has been given. They can either be "post-processed" into rotationally invariant states, or perhaps a completely different algorithm may be found which produces rotationally invariant shapes natively. Generally, restoration of symmetries broken by the shape-generating algorithm is needed whenever they are not broken by the physical ground state.

The existence of shapes provides an unexpected perspective on the fermion sign problem [17. Given that the Euler-boson wave functions are symmetric, the fermion sign problem appears only because there exists more than one shape. Conversely, if a problem could be described by the excitations of a single shape, the whole physical space of the system could be described in the Euler boson language, avoiding the sign problem. One can envisage imposing such a scenario in a Kohn-Sham-like approach, choosing a particular shape by qualitative argument, and making it give the correct binding energy with a selfconsistently derived single-particle basis. Such a program is conceptually similar to a fixed-node approach [25, 28, except that some movement of the nodes is still allowed, due to optimization in the Euler-boson sector.

The finite number of shapes brings variation and simulation closer together than is usually understood. The fact that $e^{-\tau H}$ is a general projector on the exact ground state becomes relative when generality is a finite range of possibilities, listed in advance. It is then a matter of expediency rather than principle to replace the universal projector $e^{-\tau H}$ with a specific projector in a given simulation. An explicit choice of ground-state projector turns a simulation into variational optimization.
From a practical point of view, the factorial rise in the number of shapes is somewhat unfortunate. However, problems involving strong correlations are usually local in nature, i.e. involve only a small number of electrons. Even in solid-state physics, this case is common, as attested by the remarkable popularity of locally based approaches, from finite-system studies to dynamical meanfield theory [29]. Taking $N_{\uparrow}=N_{\downarrow}=4$ as a modestly ambitious limit of practicality for $d=3$ - meaning that 255 of the 576 shapes for this case have been generated on the author's laptop, while the rest would require additional optimization and/or a bigger computer — problems with up to eight unpolarized electrons are within reach, which is competitive as of this writing. In two dimensions, the situation is naturally better.

The expression (45) collects the two main results of this work. First, there is a finite number of shapes in which any wave function can be expanded. In physical terms, there is a finite number of possible $N$-body vacua. Second, the polynomial coefficients in this expansion, or excitations of the vacua, are 1D-bosonic, i.e. symmetric in the $N$ space coordinates on each of the $d$ axes separately.

To conclude, the notion of the $N$-body vacuum in $d$ space dimensions has been given a precise and general algebraic meaning for fixed $N$ and $d$. An algorithm to construct all possible vacua was presented, and it was shown that they finitely generate the full Hilbert space of $N$ identical particles. It is hoped that these insights will lead to advances in practical calculation, at least for values of $N$ similar to those encountered in contemporary work.

\section{ACKNOWLEDGMENTS}

I thank D. Svrtan for his help and interest in this work. Conversations with O. S. Barišić, I. Batistić, and M. Primc are gratefully acknowledged. Thanks are also due to J. Ciosłowski, P.-F. Loos, J. Tahir-Kheli, H. Vančik and J. Zaanen for reading and commenting upon the manuscript. This work was supported by the Croatian Ministry of Science grant 119-1191458-0512 and by the University of Zagreb grant 202759.
[1] W. Kohn and L. J. Sham, Phys. Rev. 140, A1133 (1965)

[2] P. A. M. Dirac, Proceedings of the Royal Society of London A: Mathematical, Physical and Engineering Sciences 112, 661 (1926)

[3] J. C. Slater, Phys. Rev. 34, 1293 (1929)

[4] H. Derksen and G. Kemper, Computational Invariant Theory, Encyclopaedia of Mathematical Sciences (Springer Berlin Heidelberg, 2002).

[5] L. Euler, Comm. Acad. Petrop. 13, 64 (1741-43, 1751), see p. 80, or arxiv:0711.3656 (transl. by J. Bell), p. 15 .
Euler gets $q^{N(N+1) / 2}$ because he starts from $1 \leq n_{1}$ in Eq. (9).

[6] R. C. King and T. A. Welsh, Journal of Physics: Conference Series 30, 1 (2006)

[7] R. J. Warburton, B. T. Miller, C. S. Dürr, C. Bödefeld, K. Karrai, J. P. Kotthaus, G. Medeiros-Ribeiro, P. M. Petroff, and S. Huant, Phys. Rev. B 58, 16221 (1998)

[8] S. Kalliakos, M. Rontani, V. Pellegrini, A. Pinczuk, A. Shinga, C. Garcia, G. Goldoni, E. Molinari, L. Pfeiffer, and K. West, Solid State Communications 149, 1436 
(2009)

[9] M.-S. Miao and R. Hoffmann, Accounts of Chemical Research 47, 1311 (2014)

[10] G. E. Andrews, The Theory of Partitions (AddisonWesley, New York, 1976).

[11] F. Faà di Bruno, Einleitung in die Theorie der Binären Formen (B. G. Teubner, Leipzig, 1881).

[12] D. I. Ford, American Journal of Physics 39, 215 (1971).

[13] A. C. Aitken, Determinants and Matrices (Oliver and Boyd, Edinburgh and London, 1939).

[14] R. P. Stanley, Enumerative Combinatorics, Vol. 2 (Cambridge University Press, Cambridge, 1999).

[15] V. Bargmann, Communications on Pure and Applied Mathematics 14, 187 (1961)

[16] V. Bargmann, Communications on Pure and Applied Mathematics 20, 1 (1967)

[17] E. Y. Loh, J. E. Gubernatis, R. T. Scalettar, S. R. White, D. J. Scalapino, and R. L. Sugar, Phys. Rev. B 41, 9301 (1990)

[18] S. Reine, T. Helgaker, and R. Lindh, Wiley Interdisciplinary Reviews: Computational Molecular Science 2, $290(2012)$
[19] P. Hohenberg and W. Kohn, Phys. Rev. 136, B864 (1964).

[20] D. Ceperley, Journal of Statistical Physics 63, 1237 (1991).

[21] P.-F. Loos and D. Bressanini, The Journal of Chemical Physics 142, 214112 (2015).

[22] M. Bajdich, L. Mitas, G. Drobný, and L. K. Wagner, Phys. Rev. B 72, 075131 (2005)

[23] R. P. Stanley, Advances in Mathematics 28, 57 (1978).

[24] S. Yamanaka, K. Koizumi, Y. Kitagawa, T. Kawakami, M. Okumura, and K. Yamaguchi, International Journal of Quantum Chemistry 105, 687 (2005)

[25] D. Bressanini, Phys. Rev. B 86, 115120 (2012)

[26] J. S. Milne, "Algebraic geometry (v6.01)," (2015), available at www.jmilne.org/math/.

[27] J. Dieudonne, The American Mathematical Monthly 79, $827(1972)$

[28] V. Filinov, High Temperature 52, 615 (2014)

[29] A. Georges, G. Kotliar, W. Krauth, and M. J. Rozenberg, Rev. Mod. Phys. 68, 13 (1996)

[30] T. Živković and Z. B. Maksić, The Journal of Chemical Physics 49, 3083 (1968)

[31] L. E. McMurchie and E. R. Davidson, Journal of Computational Physics 26, 218 (1978)

\section{Appendix A: Notes on Schur functions 14}

The classic definition of Schur functions is a ratio of two determinants. The denominator is the Vandermonde determinant in some indeterminates $z_{i}$

$$
\Delta\left(z_{1}, z_{2}, \ldots, z_{N}\right) \equiv\left|\begin{array}{cccc}
z_{1}^{N-1} & z_{2}^{N-1} & \cdots & z_{N}^{N-1} \\
z_{1}^{N-2} & z_{2}^{N-2} & \cdots & z_{N}^{N-2} \\
\vdots & \vdots & & \vdots \\
z_{1} & z_{2} & \ldots & z_{N} \\
1 & 1 & \cdots & 1
\end{array}\right|=\prod_{1 \leq i<j \leq N}\left(z_{i}-z_{j}\right)
$$

which physicists would call a ground-state Slater determinant. The numerator is a similar determinant with some higher powers of the $z_{i}$ - an excited state in physicists' terms, while mathematicians sometimes call it a generalized Vandermonde determinant. If $\lambda=\left(\lambda_{1}, \ldots, \lambda_{N}\right)$ is a non-increasing sequence of natural numbers or zeros (a partition of the number $|\lambda| \equiv \lambda_{1}+\ldots+\lambda_{N}$ into at most $N$ parts), then the Schur function $s_{\lambda}$ is defined by

$$
s_{\lambda} \equiv \frac{1}{\Delta\left(z_{1}, z_{2}, \ldots, z_{N}\right)}\left|\begin{array}{cccc}
z_{1}^{N-1+\lambda_{1}} & z_{2}^{N-1+\lambda_{1}} & \ldots & z_{N-1+\lambda_{1}}^{N-1} \\
z_{1}^{N-2+\lambda_{2}} & z_{2}^{N-2+\lambda_{2}} & \ldots & z_{N}^{N-2+\lambda_{2}} \\
\vdots & \vdots & & \vdots \\
z_{1}^{\lambda_{N}} & z_{2}^{\lambda_{N}} & \ldots & z_{N}^{\lambda_{N}}
\end{array}\right| .
$$

The divisibility of the numerator by the denominator may be inferred from the fact that both vanish when any two $z_{i}=z_{j}$. The result of the division is given by a combinatorial interpretation of $s_{\lambda}$. Take a Young tableau of shape $\lambda$ and fill it with natural numbers not greater than $N$, increasing along columns and nondecreasing along rows. Call $n_{k} \geq 0$ the number of times the number $k$ appears in the tableau. A type $T(\lambda)$ is just a particular filling so obtained, for a given shape $\lambda$; then

$$
s_{\lambda}=\sum_{T(\lambda)} z_{1}^{n_{1}} z_{2}^{n_{2}} \cdots z_{N}^{n_{N}},
$$

where the sum is over all possible types. Thus coefficients in Schur functions must be natural (counting) numbers. Operationally, this formula is much simpler than the determinantal one. For example,

$$
s_{1}=z_{1}+z_{2}+\ldots+z_{N}
$$


because a single box can be filled with the numbers $1,2, \ldots, N$ only one at a time. On the other hand, if all the $\lambda_{i}=1$, this corresponds to a vertical strip of height $N$, which can be filled in only one way,

$$
s_{1^{N}}=z_{1} z_{2} \cdots z_{N}
$$

The elementary symmetric functions $e_{k}$ similarly correspond to vertical strips of height $k$ : they are the Schur functions of the partition $\lambda=(1 \ldots 1)_{\mathrm{k} \text { times }}=1^{k}$. E.g. for $k=2$ in $N=3$ variables, $s_{11}=s_{1^{2}}=z_{1} z_{2}+z_{1} z_{3}+z_{2} z_{3}$.

\section{Appendix B: Matrix element of the Coulomb force}

In quantum chemical calculations, one typically uses matrix elements between non-orthogonal Hermite Gaussian functions [30, which are best calculated recursively 31. I was not able to locate the corresponding closed expression for orthogonal Hermite functions in the literature, so I give it here, without pretense to originality.

Let

$$
\left[\vec{n} \vec{n}^{\prime}\left|V_{C}\right| \vec{m} \vec{m}^{\prime}\right]=\int d \vec{R} d \vec{R}^{\prime} \Phi_{\vec{n}}^{*}(\vec{R}) \Phi_{\vec{n}^{\prime}}^{*}\left(\vec{R}^{\prime}\right) \frac{1}{\left|\vec{R}-\vec{R}^{\prime}\right|} \Phi_{\vec{m}}(\vec{R}) \Phi_{\vec{m}^{\prime}}\left(\vec{R}^{\prime}\right)
$$

be the two-body matrix element between products of unnormalized Hermite functions,

$$
\Phi_{\vec{n}}(\vec{R}) \equiv \phi_{n_{1}}\left(R_{1}\right) \cdots \phi_{n_{d}}\left(R_{d}\right), \quad \phi_{n}(x)=H_{n}(x) e^{-x^{2} / 2},
$$

where $H_{n}$ is the Hermite polynomial. Using the standard trick [18]

$$
\frac{1}{|r|}=\frac{1}{\sqrt{\pi}} \int_{-\infty}^{+\infty} e^{-r^{2} w^{2}} d w
$$

one finds, for dimensions $d>1$ :

$$
\left[\vec{n} \vec{n}^{\prime}\left|V_{C}\right| \vec{m} \vec{m}^{\prime}\right]=\pi^{d} \sqrt{\frac{2}{\pi}} \int_{0}^{1} d w\left(1-w^{2}\right)^{(d-3) / 2} \prod_{i=1}^{d} \sum_{k_{i}=0}^{n_{i}+m_{i}} \sum_{k_{i}^{\prime}=0}^{n_{i}^{\prime}+m_{i}^{\prime}} a_{k_{i}}^{n_{i} m_{i}} a_{k_{i}^{\prime}}^{n_{i}^{\prime} m_{i}^{\prime}}(-1)^{k_{i}} H_{k_{i}+k_{i}^{\prime}}(0)\left(\frac{w}{\sqrt{2}}\right)^{k_{i}+k_{i}^{\prime}}
$$

where

$$
a_{k}^{n m}=\frac{2^{(n+m-k) / 2} n ! m !}{\left(\frac{m+n-k}{2}\right) !\left(\frac{k+n-m}{2}\right) !\left(\frac{k+m-n}{2}\right) !}
$$

for $n+m+k$ even and non-negative factorials in the denominator, zero otherwise. The Hermite polynomials $H_{k+k^{\prime}}(0)$, evaluated at zero, are zero for $k+k^{\prime}$ odd, and

$$
(-1)^{k} H_{k+k^{\prime}}(0)=(-1)^{\left(k-k^{\prime}\right) / 2} \frac{\left(k+k^{\prime}\right) !}{\left(\frac{k+k^{\prime}}{2}\right) !}
$$

for $k+k^{\prime}$ even. Finally, when the product in Eq. $\mathrm{B} 4$ is expanded, the integrals over $w$ give the beta function in place of Boys' function [18]:

$$
I_{d}(l)=\int_{0}^{1}\left(1-w^{2}\right)^{(d-3) / 2} w^{l} d w=B\left(\frac{l+1}{2}, \frac{d-1}{2}\right)=\frac{\Gamma\left(\frac{l+1}{2}\right) \Gamma\left(\frac{d-1}{2}\right)}{2 \Gamma\left(\frac{l+d}{2}\right)} .
$$

In particular,

$$
I_{2}(l)=\frac{\pi}{2^{l+1}}\left(\begin{array}{c}
l \\
l / 2
\end{array}\right) \approx \sqrt{\frac{\pi}{2 l}}, \quad I_{3}(l)=\frac{1}{l+1},
$$

noting that $l=\sum_{i} k_{i}+k_{i}^{\prime}$ is always even. 